Check for updates

Cite this: RSC Adv., 2017, 7, 34321

Received 16th May 2017

DOI: $10.1039 / c 7 r a 05538 b$

rsc.li/rsc-advances
Accepted 4th July 2017

\section{A comparative analysis of antibacterial properties and inflammatory responses for the KR-12 peptide on titanium and PEGylated titanium surfaces}

\begin{abstract}
Bin'en Nie, $\mathbb{D} \dagger^{a}$ Teng Long, $\dagger^{a}$ Hui Li, ${ }^{a}$ Xiaojie Wang ${ }^{b}$ and Bing Yue*a
Covalent immobilisation of antimicrobial peptides on titanium (Ti) surfaces has been widely performed to inhibit bacterial adhesion and biofilm formation, however, it is unclear whether a spacer is necessary in this process for anti-bacteria adhesion, while still exhibiting good biocompatibility. We investigated the antibacterial properties and inflammatory response of $\mathrm{Ti}$ and PEGylated Ti with covalently immobilized KR-12 peptide on the surfaces. The KR-12 peptide was derived from LL-37, which is bactericidal and bacteriostatic in solution. Successful covalent immobilisation was determined by $\mathrm{X}$-ray photoelectron spectrometry, contact angle measurements, and atomic force microscopy. KR-12 incorporation significantly decreased the bacterial adhesion to the Ti surface. Compared to the Ti-KR-12, the KR-12 immobilisation via a PEG spacer increased anti-microbial efficiency and reduced in vitro adhesion and biofilm formation of Staphylococcus epidermidis. Activated macrophages deprived from THP-1 cells with long filopodia were observed on the titanium surface, which was in agreement with increased TNF$\alpha$ and IL-1 $\beta$ secretion. The KR-12-modified Ti surface and PEGylated Ti surface significantly decreased TNF- $\alpha$ and IL-1 $\beta$ secretion and macrophages remained in inactivated round states. The grafting of a PEG spacer prior to KR-12 immobilisation on the Ti surface improved the antibacterial properties and reduced macrophage activation, and may also decrease overall inflammatory responses. Thus, this approach showed the potential to biofunctionalize $\mathrm{Ti}$ for anti-bacteria adhesion and reducing macrophage inflammation.
\end{abstract}

\section{Introduction}

Titanium (Ti) is widely applied in the clinic, however, due to it being bio-inert, various approaches had been applied to fabricate biofunctional Ti implants with immobilized bioactive components including hydroxylapatite (HA), silicate or strontium/sliver doped HA, antibiotics, trace elements, and antimicrobial peptides (AMPs). ${ }^{1-5}$ Among them, AMPs are a new class of antibiotics. Unlike traditional antibiotics, they have broad-spectrum antimicrobial ability with lower cytotoxicity and accordingly, they have the potential to replace the use of antibiotics. ${ }^{6}$ The use of AMP antimicrobial coatings in the biofunctionalisation of biomedical devices and Ti implants has been widely investigated, owing to their lower cytotoxicity and minimal development of pathogen resistance compared to conventional antibiotics. ${ }^{7-12}$ Various approaches based on the physical or chemical immobilisation of AMPs have been

\footnotetext{
${ }^{a}$ Department of Bone and Joint Surgery, Renji Hospital, Shanghai Jiaotong University School of Medicine, P. R. China. E-mail: advbmp2@163.com; Tel: +86-21-53882199 ${ }^{b}$ Department of General Dentistry, Ninth People's Hospital, Shanghai Jiao Tong University School of Medicine, Shanghai Key Laboratory of Stomatology, Shanghai 200011, People's Republic of China

$\dagger$ The authors contributed equally to this work.
}

developed to generate antibacterial surfaces, including covalent immobilisation, layer-by-layer techniques, and local release on the Ti surface. ${ }^{13-15}$ The local controlled release of AMPs has certain disadvantages, including short effective times, large dose requirements, and the development of bacterial resistance when small doses are used. Covalent immobilisation of AMPs on Ti surfaces is an effective approach to mitigate Ti implantassociated infections with long-term stability and low toxicity. ${ }^{16,17}$

However, it is not clear whether it is essential to tether a spacer to the Ti surface prior to the covalent immobilisation of AMPs. LL-37, the only human cationic antimicrobial peptide, is composed of 37 amino acids with a broad spectrum of antibacterial activity and participates in various immune systems, including inflammatory responses, tissue repair, and antibacterial properties. ${ }^{18}$ The application of a flexible hydrophilic poly(ethylene glycol) spacer and selective N-terminal conjugation of LL-37 results in a surface peptide layer that is capable of killing bacteria on contact. ${ }^{19}$ Thus, incorporation of a space is recommended prior to immobilisation of LL-37 on the titanium surface. ${ }^{17}$ However, some studies have suggested that covalently immobilised AMPs on Ti surfaces maintain equivalent or superior antimicrobial activity without a spacer. ${ }^{20,21}$ In one study, the application of a spacer was more effective for killing 
bacteria than the direct immobilisation of the LL-37 peptide to the titanium surface. ${ }^{22}$ The need to bind a spacer prior to covalent immobilisation of AMPs on biomedical device surfaces partially depends on the mode of action of the specific AMP. ${ }^{17}$

Polyethylene glycol (PEG) is a long chain polymer that is widely used in tissue engineering, medicine, food, owing to its high degree of hydrophilicity, water solubility, good biocompatibility and lack of toxicity. ${ }^{23-27}$ The synthetic hydrophilic polymer PEG and its derivatives are the most widely used antifouling and anti-bacterial materials. AMPs can be covalently immobilised using PEG that bears functional groups suitable for covalent peptide immobilisation. The polymer PEG can serve as an anti-protein adsorption anti-bacterial adhesion surface; it can also act as a flexible spacer for covalent immobilisation of AMP and promote AMP-bacteria interactions. ${ }^{7}$

Cytocompatibility and inflammatory responses must be considered in the biofunctionalisation of $\mathrm{Ti}^{28,29}$ Macrophage responses always influence implant performance in vivo, since tissue macrophages are among the first cells recruited around the implant. ${ }^{30}$ Macrophages can differentiate into various phenotypes and exert different functions under different micro-environments and stimuli. Macrophage polarisation is the main approach to become an activated macrophage, i.e., a classical (M1) or alternative type (M2), which is related to the infection and tissue repair. Typically, M1 macrophages are interferon-inducible (IFN- $\gamma$ ) and express pro-inflammatory cytokines (TNF- $\alpha$, IL-1 $\beta$, IL-6, and IL-8). These inflammatory cytokines can recruit other immune responsive cells, further exacerbate local inflammatory response. Unwanted inflammatory response is one of the major obstacles to osteogenesis after injury. Various studies have focused on Ti surface modifications to promote osteogenic differentiation and inhibit bacterial colonisation. ${ }^{31,32}$ However, before stem cells can arrive to the Ti implant surface to differentiate into osteoblasts and form bone, the inflammatory response must be resolved since chronic inflammation can inhibit bone-implant integration. $^{33,34}$ Therefore, controlling inflammatory responses to implants is an important issue for medical devices owing to the negative effects of chronic inflammation on device performance in vivo. Controlling inflammatory responses to implants is an important issue for medical devices owing to the negative effects of chronic inflammation on device performance in vivo. ${ }^{35}$

The KR-12 peptide was deprived from human cathelicidin LL-37, possessing both antimicrobial and anti-endotoxic activities, without mammalian cell toxicity. ${ }^{36}$ The production cost of KR-12 is much lower than that of LL-37, which makes the KR-12 peptide an excellent candidate for fabricating an antimicrobial surface for biomedical devices and Ti implants. ${ }^{37}$ In the present study, we examined whether a PEG spacer improved the antibacterial properties of Ti prior to the covalent immobilisation of KR-12. We also investigated the inflammatory response to the KR-12 peptide on Ti and PEGylated Ti surfaces. This will help us develop a suitable approach to tether the KR-12 peptide to the $\mathrm{Ti}$ surface, achieving optimal anti-bacterial effects and reduced macrophage inflammatory responses.

\section{Materials and methods}

\subsection{Materials}

The following materials were used: Ti6Al4V (Shanghai Yan Ti Metal Material Co. Ltd.), KR-12 peptide (GL BioChem, Shanghai, China; sequence, KRIVQRIKDFLR; purity, >95\%), polyethylene glycol (3.4 kD, JenKem Technology, Beijing, China), 3-aminopropyltriethoxysilane (APTES; Sinopharm Chemical Reagent Co., Shanghai, China), LIVE/DEAD® BacLight Bacterial Viability Kits (Molecular Probes, Eugene, OR, USA), 1-ethyl-3-(3-dimethylaminopropyl)carbodiimide (EDC, Tokyo, Japan), $N$-hydroxysulfosuccinimide (sulfo-NHS, Shanghai, China), LDH Cytotoxicity Kit (Beyotime Biotechnology, Haimen, China), alpha minimum essential medium $(\alpha-$ MEM; Hyclone, Logan, UT, USA), fetal bovine serum (FBS; Gibco, Australia), trypsin-EDTA (0.5\%), rhodamine phalloidin (cytoskeleton), 4',6-diamidino-2-phenylindole (DAPI; SigmaAldrich), IMMULITE®/IMMULITE® 1000 TNF- $\alpha$, and IMMULITE $® / I M M U L I T E \circledast 1000$ IL-1 $\beta$ (SIEMENS, Munich, Germany).

\subsection{Methods}

2.2.1. Covalent immobilisation of HOOC-PEG-NH $\mathrm{H}_{2}$ or/and KR-12 peptides on titanium surfaces. Ti surfaces were polished and ultrasonically cleaned in a detergent solution, acetone, and ethanol. Then, the cleaned Ti was soaked in $5 \mathrm{M} \mathrm{NaOH}$ solution at $80{ }^{\circ} \mathrm{C}$ for $24 \mathrm{~h}$ to introduce $\mathrm{OH}$ groups to the Ti surface, followed by deionized water at $80{ }^{\circ} \mathrm{C}$ for $8 \mathrm{~h}$. They were subsequently immersed in a $10 \mathrm{wt} \%$ anhydrous toluene solution of 3(2-aminoethylamino)propyltrimethoxysilane at $100{ }^{\circ} \mathrm{C}$ for $12 \mathrm{~h}$, to introduce amino groups to the Ti surface (denoted Ti-AA). For PEG immobilisation, the Ti-AA specimens were immersed in a PEG disuccinate mixture composed of $1 \mathrm{mg} \mathrm{mL}{ }^{-1}$ PEG-disuccinate of $3400 \mathrm{Da}, 20 \mathrm{mg}$ of EDC, and $15 \mathrm{mg}$ of NHS in $10 \mathrm{~mL}$ of deionized water. The reaction was carried out at $60^{\circ} \mathrm{C}$ for $4 \mathrm{~h}$, and the specimens were sonicated and rinsed with water to remove any ungrafted moieties on the surface; these samples were denoted Ti-P. For KR-12 peptide covalent immobilisation: Ti-AA substrates were incubated with KR-12 peptide solution $\left(1 \mathrm{mg} \mathrm{mL}{ }^{-1}\right)$, under the conditions of EDC and NHS. PEGylated titanium was soaked in $4 \mathrm{~mL}$ of KR-12 peptide $\left(1 \mathrm{mg} \mathrm{mL}^{-1}\right)$ and oscillated for $8 \mathrm{~h}$ at $37^{\circ} \mathrm{C}$ under the conditions of EDC and NHS to yield KR-12-conjugated PEGylated titanium (Fig. 1). It was then washed with PBS to detach the ungrafted KR-12 peptide. This composite was denoted Ti-P-KR-12. The amount of KR-12 peptide covalent on the $\mathrm{Ti}$ or PEGyalated $\mathrm{Ti}$ surface were determined with biuret reagent as precious described. ${ }^{37}$

\subsubsection{Characterisation}

$X$-ray photoelectron spectroscopy (XPS). Each step in the biofunctionalisation of Ti was characterized by XPS; briefly, the elemental composition of the surface was analysed and all energies are reported as binding energies in $\mathrm{eV}$ and referenced to the $\mathrm{C}$ 1s signal (set to $285.0 \mathrm{eV}$ ).

Atomic force microscopy (AFM). The surface topography of each $\mathrm{Ti}$ substrate was characterized using atomic force microscopy (AFM). Briefly, AFM images were obtained in the 
tapping mode at $20-25{ }^{\circ} \mathrm{C}$ in air with a Seiko SPI3800N Station (Seiko Instruments, Inc., Chiba, Japan), using silicon tips (NSG10, NT-MDT) with a resonance frequency of approximately $300 \mathrm{kHz}$. The average surface roughness values (roughness average) were also measured by AFM.

2.2.3. Water contact angle analysis. The hydrophilicity of each $\mathrm{Ti}$ substrate was measured using a contact angle meter (DMCE1, Kyowa Interface Science, Japan). Drops of ultrapure water were delivered to the substrate surface with a set drop volume of $10 \mu \mathrm{L}$ at a dosing rate of $1 \mu \mathrm{L} \mathrm{s}^{-1}$. An average of 10 readings were obtained for each sample and triplicate measurements were made for each type of Ti.

2.2.4. Bacterial colonisation on the modified titanium surface. Staphylococcus epidermidis was cultured overnight in Tryptic Soy Broth (TSB), and bacteria were re-suspended in sterile TSB to approximately $1 \times 10^{5} \mathrm{CFU} \mathrm{mL}^{-1}$. Bacteria were then added to a 24-well plate that contained various $\mathrm{Ti}$ substrates. The anti-bacterial assay was performed for the following groups: Ti, Ti-AA, Ti-P, Ti-KR-12, and Ti-P-KR-12. The Ti samples and the bacterial solution were incubated for $6 \mathrm{~h}$ and $24 \mathrm{~h}$ at $37^{\circ} \mathrm{C}$. The spread plate method, confocal laser-scanning microscopy (CLSM) and scanning electron microscopy (SEM) were used to characterize bacterial colonisation on each $\mathrm{Ti}$ sample.

For the spread method, the total number of surviving bacteria was determined by added serial dilutions of the bacteria to agar plates. Bacteria were cultured in a constant temperature incubator overnight. The total number of surviving bacteria was counted. The antibacterial rate of Ti samples was estimated as follows: $R_{\mathrm{a}}(\%)=(A-B) / A \times 100$, where $A$ represents the viable bacteria on the Ti surface and $B$ represents viable bacteria on various Ti surfaces.

CLSM was applied to characterize the live or dead bacteria on various Ti substrate surfaces. Briefly, surfaces were washed twice with PBS gently to remove non-adherent bacteria. $\mathrm{Ti}$ samples were stained with the LIVE/DEAD BacLight Bacteria Viability Kit (Molecular Probes) according to the manufacturer's instructions. The samples were visualized by CLSM (Leica TCS SP2, Germany).

For SEM, each Ti sample was fixed with $2.5 \%$ glutaraldehyde overnight, dehydrated in a graded ethanol series $(30 \%, 50 \%$, $70 \%, 80 \%$, 90\%, and $100 \%$ ) for $10 \mathrm{~min}$, freeze-dried, sputter coated with carbon, and observed by SEM (JEOL JSM-6700F, Tokyo, Japan).

2.2.5. Macrophage inflammatory responses and cytotoxicity on the titanium surface. The human macrophage cell line THP-1 was purchased from the Chinese Academy of Sciences (Shanghai, China) and maintained in RPMI 1640 medium supplemented with $10 \%$ FBS at $37{ }^{\circ} \mathrm{C}$ in a humidified $5 \% \mathrm{CO}_{2}$ incubator. Macrophages were induced with PMA at a concentration of $100 \mathrm{ng} \mathrm{mL} \mathrm{m}^{-1}$ for $24 \mathrm{~h}$ and cultured for $24 \mathrm{~h}$ in blank media (10\% FBS). The cell density was adjusted to $1 \times 10^{5}$ per $\mathrm{mL}$. Ti substrates were plated on a 24 -well plate prior to seed 1 $\mathrm{mL}$ cell suspension at a density of $1 \times 10^{5}$ per $\mathrm{mL}$. After $24 \mathrm{~h}$ and
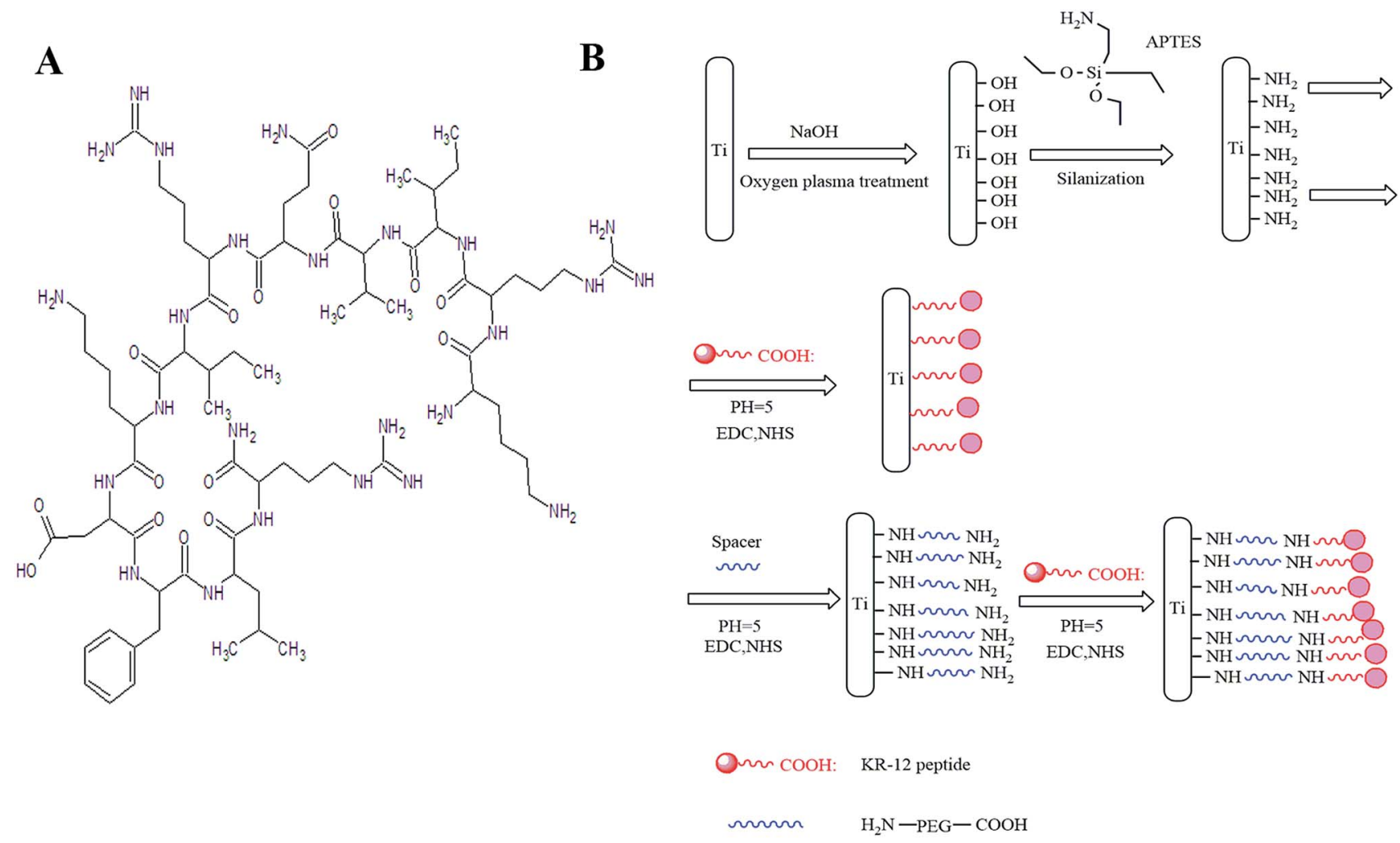

Fig. 1 (A) Chemical structure of the KR-12 peptide. (B) Diagrammatic sketch of the covalent immobilisation of the KR-12 peptide on the PEGylated Ti surface. 
$48 \mathrm{~h}$ of incubation, the levels of the pro-inflammatory cytokines (TNF- $\alpha$ and IL-1 $\beta$ ) in the cell supernatant was determined using IMMULITE/IMMULITE 1000 TNF- $\alpha$ and IL-1 $\beta$ Kits respectively (Siemens Healthcare Diagnostics, Inc.), according to the manufacturer's instructions. The detection limits of the assays were 0.12-1000 pg mL ${ }^{-1}$ for TNF- $\alpha, 0.70-1000 \mathrm{pg} \mathrm{mL}{ }^{-1}$ for IL-6, 2-7500 $\mathrm{pg} \mathrm{mL} \mathrm{m}^{-1}$ for IL-8, and $1.5-1000 \mathrm{pg} \mathrm{mL}{ }^{-1}$ for IL-1 $\beta$. The release of lactate dehydrogenase ( $\mathrm{LDH})$ enzyme in the supernatant was used to investigate cytotoxicity using the Cytotoxicity Detection Kit. LDH activity was determined spectrophotometrically at $490 \mathrm{~nm}$.

2.2.6. CLSM to characterise the macrophage morphology. The macrophage morphology and cytoskeletons were characterized by CLSM. After $24 \mathrm{~h}$ and $48 \mathrm{~h}$ of incubation with THP-1deprived macrophages, the Ti substrate was washed with PBS to remove non-adherent cells and fixed with $4 \%$ paraformaldehyde overnight. Cells attached on $\mathrm{Ti}$ samples, including the Ti substrate, Ti-AA, Ti-P, Ti-KR-12, and Ti-P-KR-12 substrates were fixed with $4 \%$ paraformaldehyde and then incubated with $0.1 \%$ Triton X-100 for $15 \mathrm{~min}$, gently washed with PBS, and then soaked in DAPI solution $\left(5 \mu \mathrm{g} \mathrm{mL}{ }^{-1}\right)$ for $15 \mathrm{~min}$ for nuclear staining. Cytoskeleton was stained with fluorescent phalloidin for $30 \mathrm{~min}$ according to the manufacturer's protocol, and the macrophage cell morphology was visualized by CLSM (Leica TCS SP2).

2.2.7. SEM to characterize the filopodia of macrophages on each Ti substrate. Macrophages were cultured as described in Section 2.2.7. Macrophage parapodia on various Ti surfaces were characterized by SEM. Briefly, at the prescribed time points, i.e., $24 \mathrm{~h}$ and $48 \mathrm{~h}$, samples were fixed with $2.5 \%$ glutaraldehyde overnight, dehydrated in a graded ethanol series (30\%, 50\%, 70\%, 80\%, 90\%, and $100 \%$ ) for $10 \mathrm{~min}$, freeze-dried, and sputter coated with carbon. Filopodia were observed by SEM (JEOL JSM-6700F).

2.2.8. Statistical analysis. All data were analysed using SPSS (version 19.0). One-way ANOVA followed by an S-N-K-test was used to evaluate differences between groups. Statistical significance was set at $P<0.05$.

\section{Results}

\subsection{Surface characterisation was analysed by XPS}

Elemental composition of silanised $\mathrm{Ti}$ samples indicated increased $\mathrm{N} \%$ and $\mathrm{C} \%$ to $3.58 \%$ and $24.25 \%$ and decreased $\mathrm{O} \%$ to $51.14 \%$. The subsequent addition of covalent PEG on the amine-functionalized surface showed an increase in $\mathrm{N} \%$ and C\% to $3.62 \%$ and $24.41 \%$. After covalent immobilisation of the KR-12 peptide on the amine-functionalised Ti surface, $\mathrm{N} \%$ and C\% increased to $4.34 \%$ and $24.13 \%$. After covalent immobilisation of the KR-12 peptide on the PEGylated Ti surface, N\% and $\mathrm{C} \%$ increased to 5.36 and 78.85 (Table 1). C 1s highresolution XPS spectra were obtained (Fig. 2). The native C 1s spectrum of Ti-P showed two peaks at binding energies of 288.1 and $284.1 \mathrm{eV}$, corresponding to $\mathrm{C}=\mathrm{O} / \mathrm{O}=\mathrm{C}-\mathrm{N}$ and $\mathrm{C}-\mathrm{C}$, which indicated that PEG was covalently attached to the aminefunctionalised surface via the reaction of $-\mathrm{COOH}$ in PEG and $-\mathrm{NH}_{2}$ on Ti-AA and formed a new amide bond. Ti-KR-12 showed two peaks at binding energies of approximately 284.4 (aliphatic
Table 1 Chemical composition and relative atomic ratios (atomic percentages, \%) of Ti, Ti-AA, Ti-P, Ti-KR-12 and Ti-P-KR-12

\begin{tabular}{lrrrrr}
\hline & \multicolumn{1}{c}{ Ti } & Ti-AA & \multicolumn{1}{c}{ Ti-P } & Ti-KR-12 & Ti-P-KR-12 \\
\hline C (\%) & 9.19 & 24.25 & 24.41 & 24.13 & 78.85 \\
N (\%) & 0.97 & 3.58 & 3.62 & 4.34 & 5.36 \\
O (\%) & 65.28 & 51.14 & 50.67 & 48.76 & 11.41 \\
Si (\%) & 4.77 & 4.51 & 5.32 & 7.46 & 0.81 \\
Ti (\%) & 17.92 & 16.04 & 15.97 & 14.81 & 0.2 \\
\hline
\end{tabular}

C-C), 258.8 eV (C-N groups), 286.1 (C-O groups) and 288.1 (C= $\mathrm{O} / \mathrm{O}=\mathrm{C}-\mathrm{N})$. Peptide immobilisation via APTES silanisation resulted in a significant increase in the high-energy component of $\mathrm{C} 1 \mathrm{~s}$ compared to controls. Ti-P-KR-12 also showed two peaks at approximately $259.8(\mathrm{C}-\mathrm{O})$ and $288.2 \mathrm{eV}(\mathrm{C}=\mathrm{O} / \mathrm{O}=\mathrm{C}-\mathrm{N})$ and an increase of $\mathrm{C}$ and $\mathrm{N}$ elemental composition compared to Ti-P and Ti-KR-12. The XPS data confirmed the covalent immobilization of PEG, KR-12, and both PEG and KR-12 on the Ti surface. The amount of KR-12 covalent bond on the Ti and PEGyalated Ti showed no significant difference.

\subsection{AFM to characterize the surface topography of each Ti sample}

The surface morphology of samples was analysed using AFM after each step of $\mathrm{Ti}$ biofunctionalisation, and changes in the $\mathrm{Ti}$ surface topography were detected. The root mean square (RMS) surface roughness for each sample was shown in Fig. 3. The roughness values were $1002.6 \pm 12 \mathrm{~nm}, 509 \pm 8 \mathrm{~nm}, 516 \pm$ $5 \mathrm{~nm}, 522 \pm 19 \mathrm{~nm}$, and $528 \pm 14 \mathrm{~nm}$ for Ti, Ti-AA, Ti-P, Ti-KR12, and Ti-P-KR-12, respectively. Ti samples pre-treated with $\mathrm{NaOH}$ etching and APTES showed a significant increase in roughness in comparison with control samples. For covalent PEG or and KR-12 on the Ti surface, the roughness was not significantly different compared with Ti-AA.

\subsection{Water contact angle}

Next, we characterised the surface contact angle of each $\mathrm{Ti}$ sample. The water contact angle for Ti, Ti-AA, Ti-P, Ti-KR-12 and Ti-P-KR-12 were $73.92^{\circ} \pm 2.41^{\circ}, 38.9^{\circ} \pm 4.07^{\circ}, 28.0^{\circ} \pm 1.38^{\circ}$, $30.7^{\circ} \pm 2.05^{\circ}, 30.4^{\circ} \pm 1.12^{\circ}$ respectively. Ti-AA decreased the contact angle values with a significant increase in hydrophilicity (Fig. 4). For Ti-P, since PEG is a highly hydrophilic polymer, when it was covalently attached to the amine-functionalised surface, it can further increase the hydrophilicity of Ti-AA.

\subsection{Bacteria colonisation on each Ti surface}

The adhesion of $S$. epidermidis to various Ti surfaces was evaluated using the spread plate method, CLSM, and SEM. Untreated Ti and Ti-AA surfaces showed large bacterial colonisation areas and high relative proportions of bacteria. PEGylated $\mathrm{Ti}$ can reduce bacteria adhesion and minimize bacterial colonisation on the surface at $6 \mathrm{~h}$. After $24 \mathrm{~h}$, the bacteria counts showed no significant differences between Ti, Ti-AA, and Ti-P. At each time point, Ti control exhibited highest number of bacterial colonies, fewer bacterial colonies were observed in Ti- 

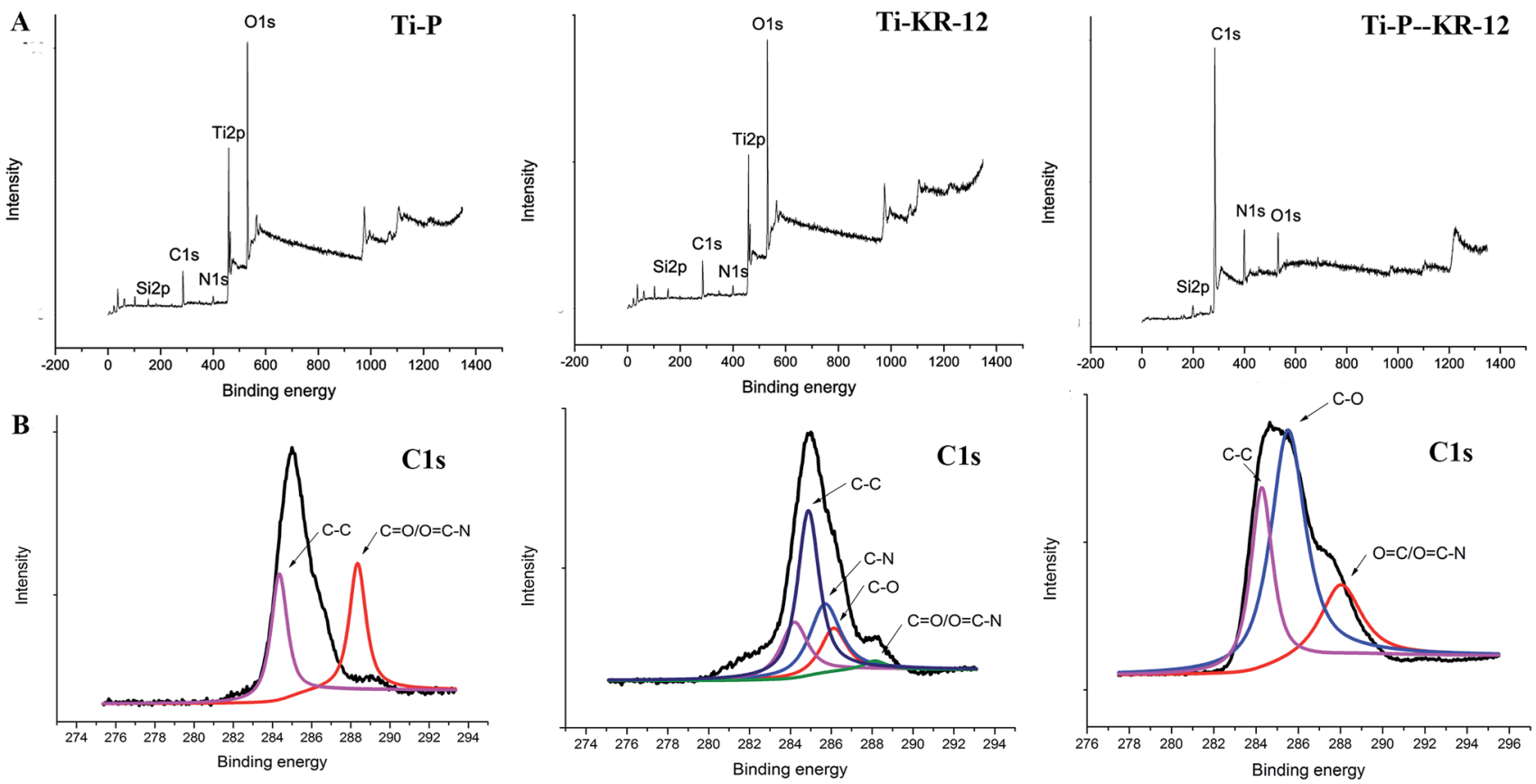

Fig. 2 XPS to characterize the successfully covalent immobilization. (A) XPS wide scan spectra of Ti-P, TiKR-12 and Ti-P-KR-12 and (B) XPS high resolution of carbon spectra signal of Ti-P, Ti-KR-12, and Ti-P-KR-12.

KR-12 and Ti-P-KR-12, while least number of bacterial colonies even were observed in Ti-P-KR-12 (Fig. 5A). Based on a quantitative analysis, Ti-KR-12 exhibited $89 \%$ bacterial inhibition, and Ti-P-KR-12 exhibited a 96\% inhibition of bacteria (Fig. 5B).

The results of live/dead bacterial staining are summarised in Fig. 6. After culturing for $6 \mathrm{~h}$, there were large amounts of viable bacteria in the $\mathrm{Ti}$ and $\mathrm{Ti}-\mathrm{AA}$ groups. Other groups demonstrated decreased the viable bacterial colonies, (green fluorescence) and increased dead bacterial colonies (red fluorescence). After $24 \mathrm{~h}$ of culture, a large amount of viable bacteria were detected in the Ti, Ti-AA, and Ti-P groups (Fig. 6A), while many dead bacteria (red) occurred in Ti-P-12. A similar trend was observed using SEM. After $6 \mathrm{~h}$ and $24 \mathrm{~h}$, there were large amounts of bacteria on the Ti, Ti-AA, and Ti-P groups, but few adherent bacteria on Ti-KR-12 and Ti-P-KR-12 (Fig. 6B).

\subsection{Inflammatory cytokine secretion}

The levels of the pro-inflammatory factors TNF- $\alpha$ and IL- $1 \beta$ in cell culture supernatant were investigated. As shown in Fig. 7A, after
$24 \mathrm{~h}$ and $48 \mathrm{~h}$, highest amount of TNF- $\alpha$ was released by cells on control Ti. After $48 \mathrm{~h}$, lowest level of TNF- $\alpha$ levels were observed in Ti-P and Ti-P-KR-12 group, which has significantly lower TNF$\alpha$ level compared to Ti and Ti-AA group $(P<0.05)$ (Fig. 7A). After $24 \mathrm{~h}$ and $48 \mathrm{~h}$, IL-1 $\beta$ was reduced on Ti-P and Ti-P-KR-12 in comparison to the Ti, Ti-AA, and Ti-KR-12 groups $(P<0.05)$. The IL-1 $\beta$ levels were significantly lower on Ti-KR-12 compared with the other Ti substrates at each time point $(P<0.05)$ (Fig. 7B).

\subsection{LDH activity on each Ti surface}

Cytotoxicity was investigated by LDH activity in the culture medium after $24 \mathrm{~h}$ and $48 \mathrm{~h}$ of incubation with macrophages, as shown in Fig. 8. Although there was a general trend of increase in LDH activity from $24 \mathrm{~h}$ to $48 \mathrm{~h}$, we did not observe any significant difference among all groups at each time point.

\subsection{Macrophage morphology based on CLSM}

The surface Ti treatment resulted in significant changes on macrophage morphology. Macrophages on $\mathrm{Ti}$ showed
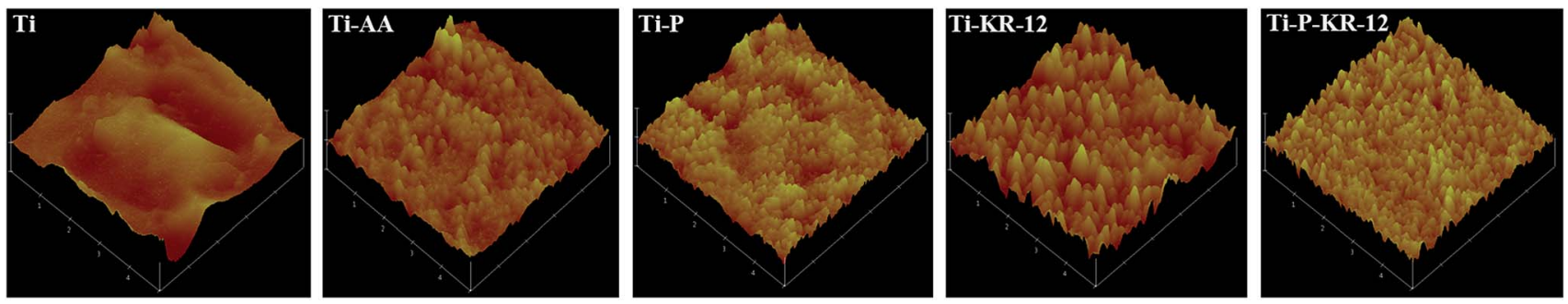

Fig. 3 AFM 3D images of the Ti surfaces. 

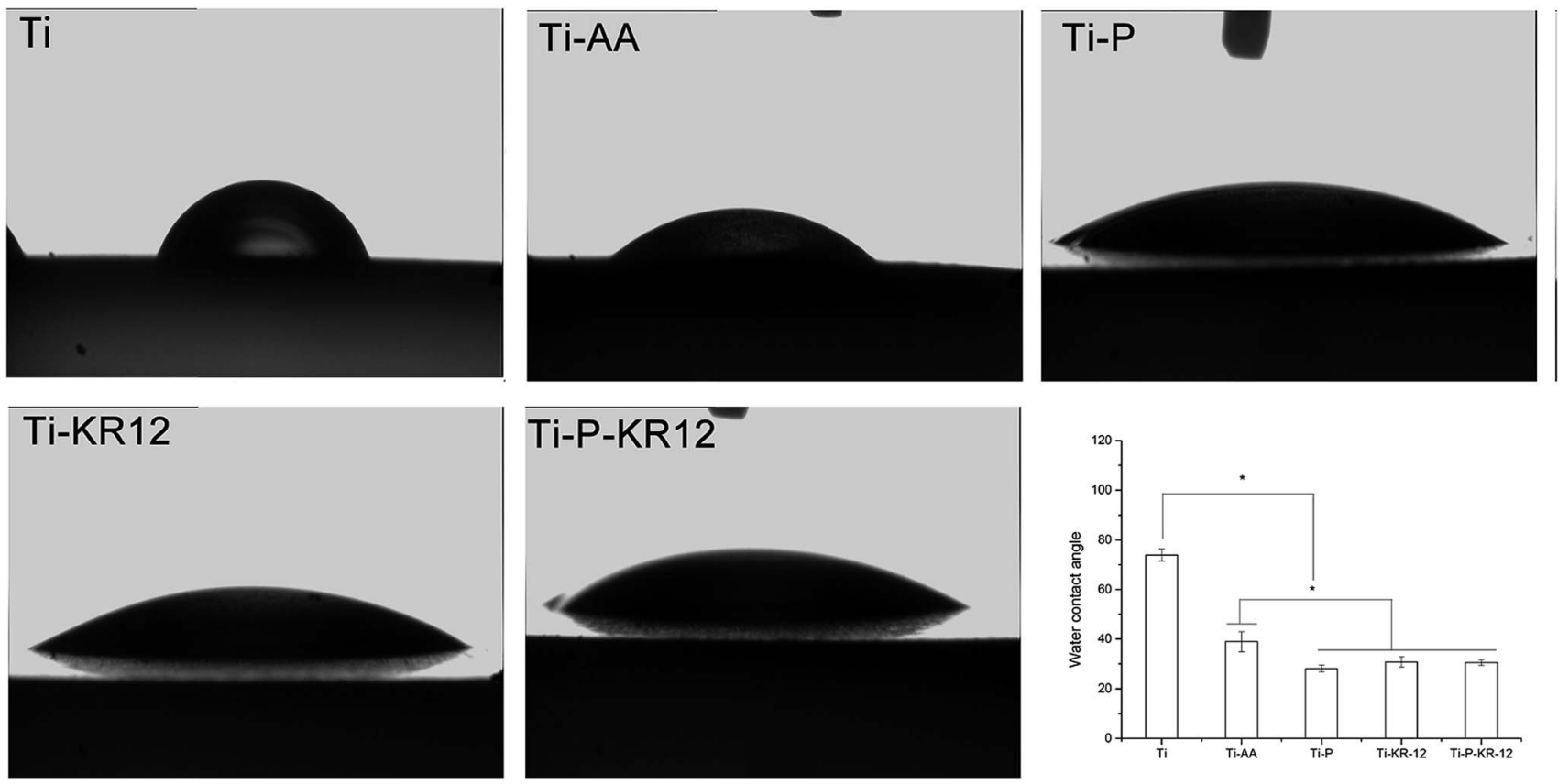

Fig. 4 Water contact angle images of each Ti sample and a quantitative characterisation. The Ti-P-KR-12 substrate was more hydrophilic than those of the other four groups. $* P<0.05$.

a larger spreading area with irregular shapes (Fig. 9). In the Ti-AA group, fewer macrophages displayed irregular shapes compared with the Ti group (Fig. 9). In the Ti-P, Ti-KR-12, and Ti-P-KR-12 groups, macrophages were almost all round in shape, indicating that the macrophages were not activated.

\subsection{SEM to characterise filopodia of macrophages on various Ti surfaces}

Macrophages on the Ti surface displayed a number of long filopodia, and cells appeared to spread more after $24 \mathrm{~h}$ and $48 \mathrm{~h}$ of co-culture in comparison to all other surfaces (i.e., Ti-AA, TiP, Ti-KR-12, and Ti-P-KR-12) (Fig. 10). In the Ti-P, Ti-KR-12, and Ti-P-KR-12 groups, macrophages maintained round shapes with shorter filopodia.

\section{Discussion}

Early implant failure can be caused by bacterial infections acquired either during or immediately after surgery. Infection remains one of the major barriers to the long-term use of medical devices in patients. In severe cases, additional surgeries are required to treat implant related infections, which are complex and resource intensive. Antibacterial coating on implants therefore provide an attractive alternative to prevent implant infection. In this study, we covalently immobilised the KR-12 peptide on the PEGylated titanium surface, which improved the antibacterial effect and biocompatibility of $\mathrm{Ti}$ surfaces.

Silanisation with APTES is an effective approach to introduce amine groups on the Ti surface, which provided binding sites for biomolecules. ${ }^{38,39} \mathrm{Ti}$ was first etched by $\mathrm{NaOH}$ to introduce
A Ti

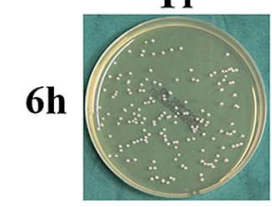

24h

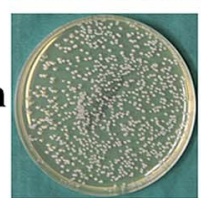

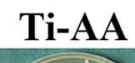
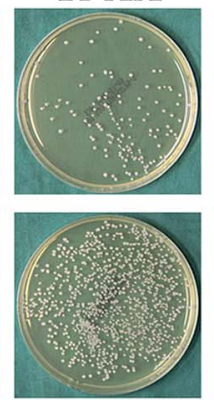

\section{Ti-P}
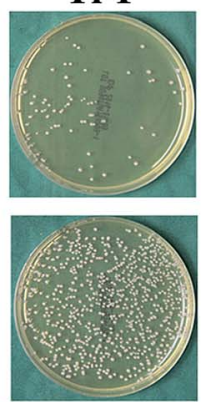
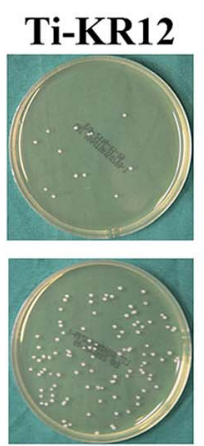
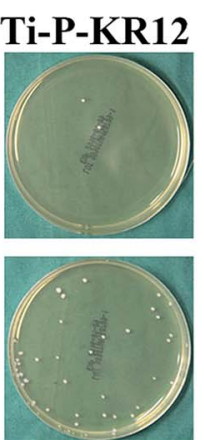

B

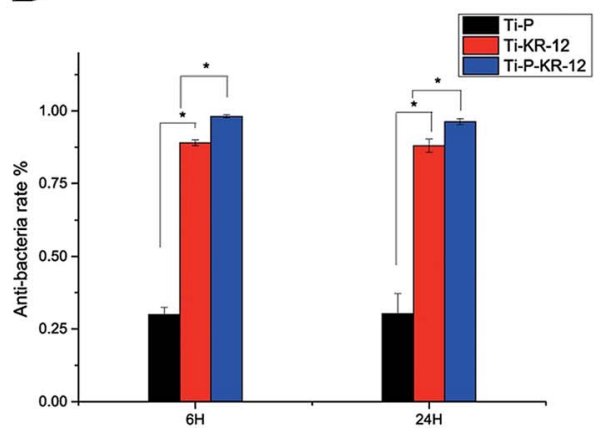

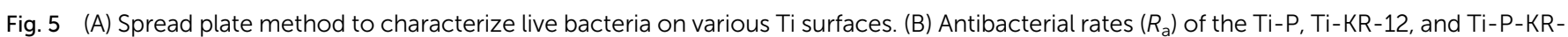
12 substrates against Staphylococcus epidermidis. Data $=$ means \pm standard error of the mean. ${ }^{*} P<0.05$. 


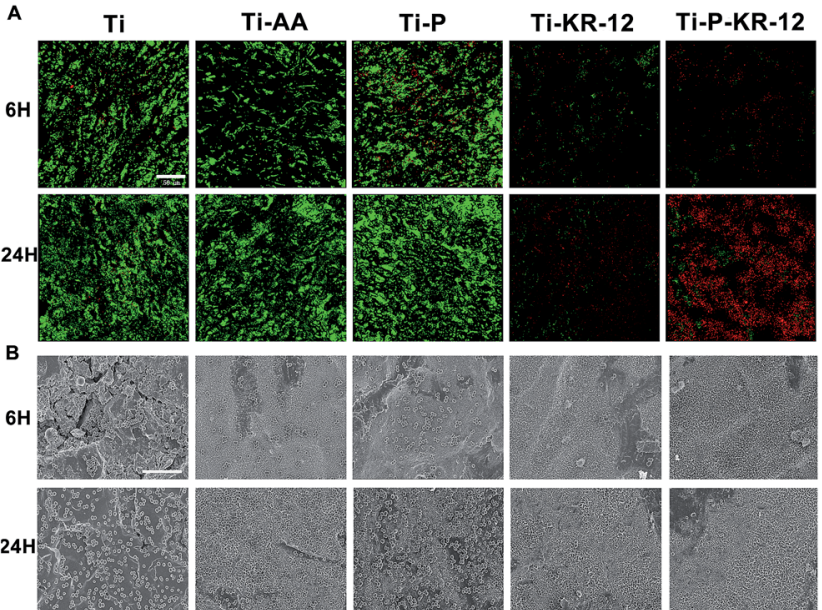

Fig. 6 Antibacterial properties of various Ti samples after $6 \mathrm{~h}$ and $24 \mathrm{~h}$ of incubation with S. epidermidis. (A) Live/dead staining; viable bacteria were stained green and dead bacteria were stained red. The scale bar is $50 \mu \mathrm{m}$. (B) SEM images of various Ti surfaces incubated with S. epidermidis after $6 \mathrm{~h}$ and $24 \mathrm{~h}$. The scale bar is $10 \mu \mathrm{m}$.

$\mathrm{OH}$ groups on the surface. In the subsequent silanisation process, $\mathrm{Si}-\mathrm{OH}$ in APTES reagent reacted with the hydroxyl on titanium and $\mathrm{Ti}-\mathrm{O}-\mathrm{Si}$, forming the amine-containing organic transition layer. Finally, biological macromolecules were immersed in solutions and fixed on the surface of the titanium surface.

As stated earlier, infection poses a major risk associated with Ti based biomedical devices. Bacteria can adhere to Ti surface and form biofilm after surgery and invade the surrounding tissue. $^{40}$ Antibacterial $\mathrm{Ti}$ coating is the main prophylaxis approach to prevent infection associated with Ti implant. ${ }^{41}$ In our study, we covalently immobilised a KR-12 peptide on the Ti surface. We observed an antibacterial rate of $89 \%$ (Fig. 5B). Ti-P possessed a PEGylated surface, which could prevent early bacterial adhesion. However, bacteria count did not differ significantly from unmodified Ti surface after $24 \mathrm{~h}$. We further

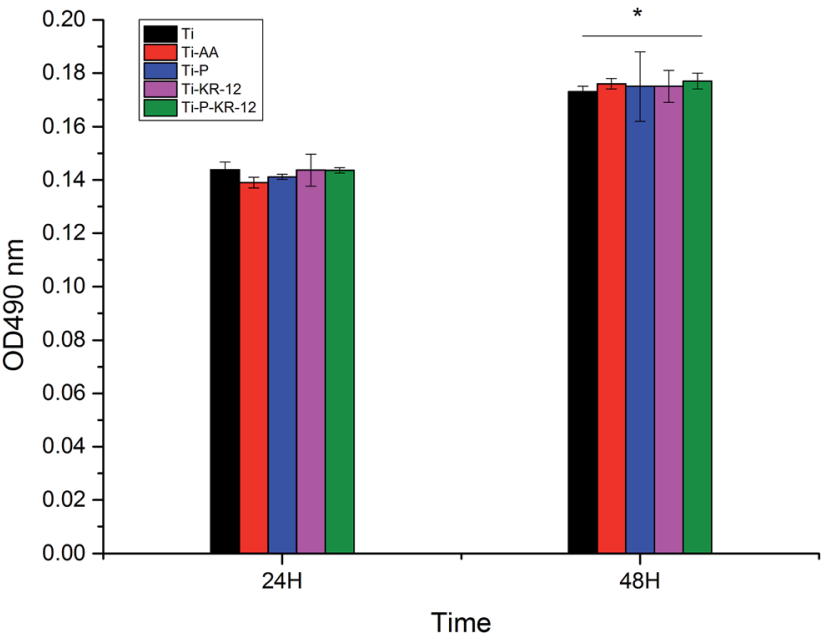

Fig. 8 Cytotoxicity of macrophages on various titanium surfaces. Cell viability, which was assessed using LDH assays, demonstrated no observable changes after $24 \mathrm{~h}$, but LDH activity was higher after $48 \mathrm{~h}$, although there were no significant differences between groups; $* P<$ 0.05 .

investigated whether Ti-P could improve the antibacterial effect when a spacer was introduced prior to KR-12 peptide binding. The antibacterial rate increased by $7 \%$ compared to the control Ti surface with the KR-12 peptide. Our results indicated that the KR-12 peptide could retain its antibacterial properties, even when covalently immobilised on the Ti surface, and the addition of a PEG spacer prior to covalent attachment of KR-12 on the Ti surface could further improve the antibacterial property. APTES itself can act as a bridge to binding bioactive molecules. On one hand, the $\mathrm{Si}-\mathrm{OH}$ can react with $\mathrm{Ti}-\mathrm{OH}$ (Ti functionalized with oxhydryl) to form $\mathrm{Ti}-\mathrm{O}-\mathrm{Si}-\mathrm{NH}_{2}$ on the Ti surface, on the other hand, the $\mathrm{Si}-\mathrm{OH}$ can react with themselves to form $\mathrm{Si}-\mathrm{O}-$ $\mathrm{Si}$ and can form a stable group with more amines on the $\mathrm{Ti}$ surface, APTES is similar to the role of bridges that could allow the mobility of the bound KR-12 peptide and preserve the antibacterial property. PEG can further increase the length of
A

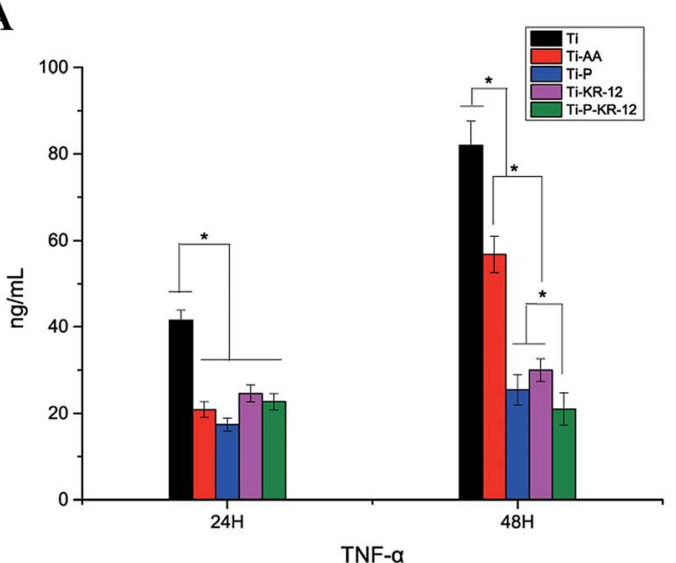

B

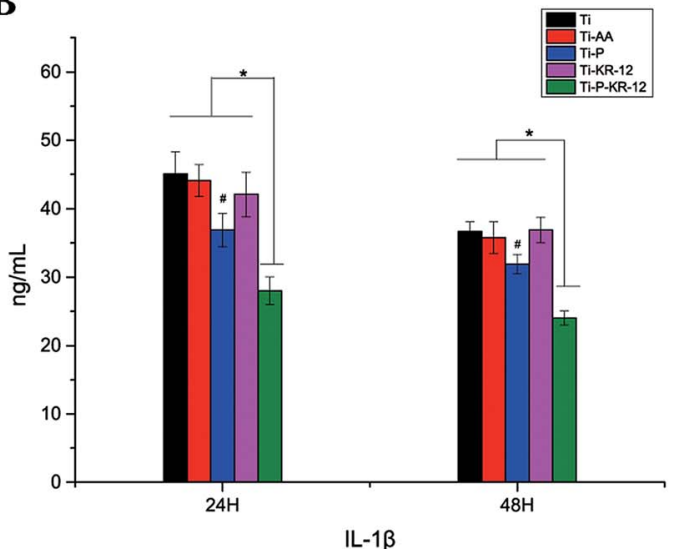

Fig. 7 (A) TNF- $\alpha$ and (B) IL-1 $\beta$ production by THP-1-deprived macrophages incubated with different Ti samples after 24 and 48 h. Data are expressed as means + SEM of three independent experiments, $* P<0.05$ 

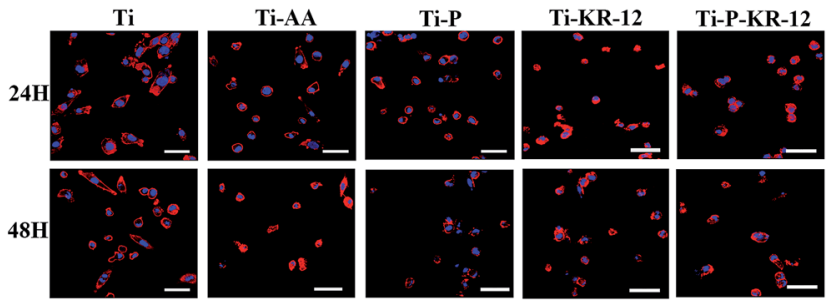

Fig. 9 Cytoskeletons of THP-1-deprived macrophages seeded on various samples were stained with rhodamine phalloidin (red), and nuclei were stained with DAPI (blue) at the indicated time points (24 and $48 \mathrm{~h}$ ). The scar bar is $50 \mu \mathrm{m}$.

spacers or directly act as a spacer for KR-12 peptide binding, which makes the spacer more flexible. As a result, KR-12 binding on PEG improves mobility and antibacterial activity compared with KR-12 binding.

As with any implants, functional modifications of titanium substrates should not pose negative effect on its biocompatibility. Implants with poor biocompatibility often triggers undesirable immune responses, such as heightened inflammation and scar healing response instead of tissue regeneration. ${ }^{42}$ The behaviour of macrophages is a useful indicator of the biocompatibility of $\mathrm{Ti}$ implants. Secretion of inflammatory cytokine and macrophage morphology can indicate the activated or non-activated state of macrophages. Activated macrophages exhibit a number of long filopodia and exhibit a larger cell spreading area, which is a hallmark of M1 phenotype. M1 macrophage is considered pro-inflammatory, which has upregulated expression of STAT1 and NF-kB and enhanced secretion of pro-inflammatory cytokines, such as TNF- $\alpha$, IL-1 $\beta$, IL-6, and IL-8. PEG is an alternative polymer that can be used to improve the hydrophilicity and biocompatibility of Ti. ${ }^{43-46}$

Unlike smooth Ti induced inflammatory macrophage activation, as indicated by increased levels of the interleukins TNF$\alpha$ and IL-1 $\beta$, the spreading area and the filopodia of macrophage on Ti, the KR-12-modified PEGylated Ti could improve the biocompatibility of $\mathrm{Ti}$, which resulted in decreased inflammatory response from macrophages. We also investigated whether

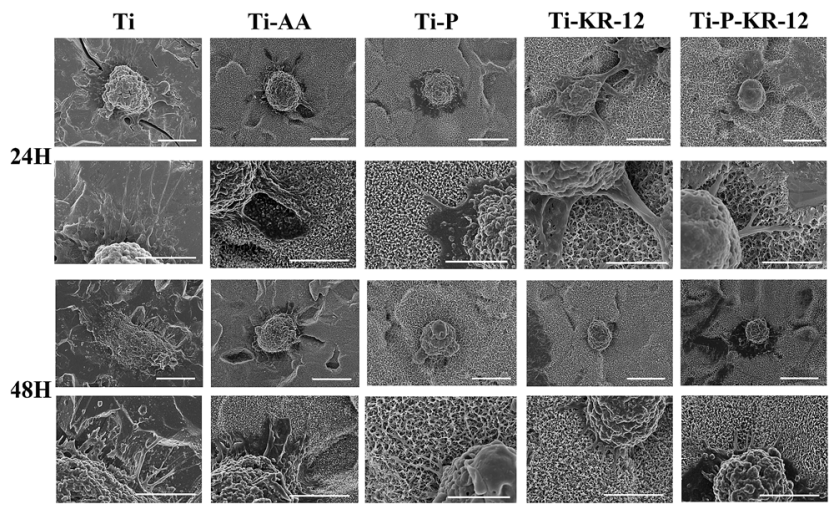

Fig. 10 Macrophage behaviour on different surfaces (SEM images), particularly with respect to the number of filopodia and level of cell spreading on the Ti, Ti-AA, Ti-P, Ti-KR-12 and Ti-P-KR-12 substrate. changes in inflammatory cytokines were caused by cytotoxicity on the various surfaces; after $24 \mathrm{~h}$ and $48 \mathrm{~h}$, the LDH activity did not differ significantly among groups, indicating that changes in inflammatory cytokines on various $\mathrm{Ti}$ surfaces were not related to changes in macrophage viability (Fig. 8). The surface characteristics of $\mathrm{Ti}$ (including the topography and wettability) can influence the behaviour of macrophages. Specifically, the hydrophilic Ti surfaces showed greater inhibition of antiinflammatory cell responses compared to hydrophobic surfaces ${ }^{47}$ In our study, Ti-AA, Ti-P, Ti-KR-12, and Ti-P-KR-12 all showed higher hydrophilicity and surface roughness than $\mathrm{Ti}$ (Fig. 3 and 4). PEG itself is a hydrophilic polymer and KR-12 showed anti-inflammatory effects in vitro ;6 $^{36}$ covalent immobilisation of PEG and KR-12 on the Ti surface can further decreased inflammatory cytokine secretion, indicating that PEG and KR12 could maintain the anti-inflammatory effects when covalently attached to the Ti surface. Furthermore, PEG could act as a flexible spacer to bind KR-12 on the Ti surface, which enhanced the antibacterial property compared with the simple binding of KR-12 on the Ti surface. The anti-inflammatory effect was higher compared to the simple PEG and KR-12 modification on Ti. Thus, KR-12 peptide-modified PEGylated $\mathrm{Ti}$ is a promising candidate for fabricating an antimicrobial Ti with good biocompatibility.

\section{Conclusion}

For the first time, we covalently immobilised the KR-12 peptide on Ti surface using a PEG spacer. With this spacer, KR-12 killed bacteria more effectively compared with the surface lacking the spacer. Additionally, macrophage activation was reduced on TiP-KR-12 compared to that on flat Ti. Our results provided a promising approach to the inhibition of bacterial colonisation on Ti surfaces and macrophage inflammation, indicating the potential to prevent peri-prosthetic infection and periimplantitis.

\section{Conflict of interest}

The authors declare no competing interests linked to this study.

\section{Authors' contributions}

Bin'en Nie, Teng Long, Hui Li and Xiaojie Wang carried out the experiments. Bin'en Nie wrote the manuscript. Bing Yue and Teng Long designed the experiments. Bing Yue revised the manuscript. All authors reviewed the manuscript.

\section{Acknowledgements}

This research was supported by National Natural Science Foundation of China (81472119, 81501856, 81501855 and 81672196), Shanghai municipal education commissionGaofeng clinical medicine grant support (20161423), and Shanghai Sailing Program (15YF1407000). 


\section{References}

1 Y. Huang, Z. Xu, X. Zhang, X. Chang, X. Zhang, Y. Li, T. Ye, R. Han, S. Han, Y. Gao, X. Du and H. Yang, J. Alloys Compd., 2017, 697, 182-199.

2 Y. Huang, X. Zhang, H. Zhang, H. Qiao, X. Zhang, T. Jia, S. Han, Y. Gao, H. Xiao and H. Yang, Ceram. Int., 2017, 43, 992-1007.

3 M. Sartori, G. Giavaresi, A. Parrilli, A. Ferrari, N. N. Aldini, M. Morra, C. Cassinelli, D. Bollati and M. Fini, International orthopaedics, 2015, 39, 2041-2052.

4 S. B. Goodman, Z. Yao, M. Keeney and F. Yang, Biomaterials, 2013, 34, 3174-3183.

5 G. Jin, H. Cao, Y. Qiao, F. Meng, H. Zhu and X. Liu, Colloids Surf., B, 2014, 117, 158-165.

6 R. E. Hancock and H. G. Sahl, Nat. Biotechnol., 2006, 24, 1551-1557.

7 S. A. Onaizi and S. S. J. Leong, Biotechnol. Adv., 2011, 29, 6774.

8 A. P. Gomes, J. F. Mano, J. A. Queiroz and I. C. Gouveia, Carbohydr. Polym., 2015, 127, 451-461.

9 L. Zhou, Y. Lai, W. Huang, S. Huang, Z. Xu, J. Chen and D. Wu, Colloids Surf., B, 2015, 128, 552-560.

10 M. Kazemzadeh-Narbat, B. F. L. Lai, C. Ding, J. N. Kizhakkedathu, R. E. W. Hancock and R. Wang, Biomaterials, 2013, 34, 5969-5977.

11 R. Chen, M. D. P. Willcox, K. K. K. Ho, D. Smyth and N. Kumar, Biomaterials, 2016, 85, 142-151.

12 A. Rai, S. Pinto, M. B. Evangelista, H. Gil, S. Kallip, M. G. S. Ferreira and L. Ferreira, Acta Biomater., 2016, 33, $64-77$.

13 J. Shi, Y. Liu, Y. Wang, J. Zhang, S. Zhao and G. Yang, Sci. Rep., 2015, 5, 16336.

14 M. Kazemzadeh-Narbat, J. Kindrachuk, K. Duan, H. Jenssen, R. E. W. Hancock and R. Wang, Biomaterials, 2010, 31, 95199526.

15 M. Kazemzadeh-Narbat, S. Noordin, B. A. Masri, D. S. Garbuz, C. P. Duncan, R. E. W. Hancock and R. Wang, J. Biomed. Mater. Res., Part B, 2012, 100, 13441352.

16 M. Godoy-Gallardo, C. Mas-Moruno, M. C. FernándezCalderón, C. Pérez-Giraldo, J. M. Manero, F. Albericio, F. J. Gil and D. Rodríguez, Acta Biomater., 2014, 10, 3522-3534.

17 F. Costa, I. F. Carvalho, R. C. Montelaro, P. Gomes and M. C. L. Martins, Acta Biomater., 2011, 7, 1431-1440.

18 K. Kuroda, K. Okumura, H. Isogai and E. Isogai, Front. Oncol., 2015, 5, 10.

19 M. Gabriel, K. Nazmi, E. C. Veerman, A. V. N. Amerongen and A. Zentner, Bioconjugate Chem., 2006, 17, 548-550.

20 X. W. Tan, T. W. Goh, P. Saraswathi, C. L. Nyein, M. Setiawan, A. Riau, R. Lakshminarayanan, S. Liu, D. Tan, R. W. Beuerman and J. S. Mehta, Antimicrob. Agents Chemother., 2014, 58, 5229-5238.

21 X. Chen, H. Hirt, Y. P. Li, S. U. Gorr and C. Aparicio, Plos One, 2014, 9, 8.
22 M. Gabriel, K. Nazmi, E. C. Veerman, A. V. Nieuw Amerongen and A. Zentner, Bioconjugate Chem., 2006, 17, 548-550.

23 J. Chen, J. Cao, J. Wang, M. F. Maitz, L. Guo, Y. Zhao, Q. Li, K. Xiong and N. Huang, J. Colloid Interface Sci., 2012, 368, 636-647.

24 J. Zhou, S. Hu, J. Ding, J. Xu, J. Shi and N. Dong, Biomedical engineering online, 2013, $12,87$.

25 Y. Tanaka, Y. Matsuo, T. Komiya, Y. Tsutsumi, H. Doi, T. Yoneyama and T. Hanawa, J. Biomed. Mater. Res., Part A, 2010, 92, 350-358.

26 J. Zhou, B. Nie, Z. Zhu, J. Ding, W. Yang, J. Shi, X. Dong, J. $\mathrm{Xu}$ and N. Dong, Biomed. Mater., 2015, 10, 065014.

27 B. e. Nie, T. Long, H. Ao, J. Zhou, T. Tang and B. Yue, Antimicrob. Agents Chemother., 2017, 61, e01766-16.

28 D. W. Xu, W. H. Yang, Y. Hu, Z. Luo, J. H. Li, Y. H. Hou, Y. Liu and K. Y. Cai, Colloids Surf., B, 2013, 110, 225235.

29 B. Nie, H. Ao, J. Zhou, T. Tang and B. Yue, Colloids Surf., B, 2016, 145, 728-739.

30 J. Kzhyshkowska, A. Gudima, V. Riabov, C. Dollinger, P. Lavalle and N. E. Vrana, J. Leukocyte Biol., 2015, 98, 953962.

31 S. W. Lee, H. J. Lee, J. W. Lee, K.-H. Kim, J.-H. Kang, M. H. Lee and S. C. Lee, Colloids Surf., B, 2015, 135, 565574.

32 E.-C. Kim, T.-H. Kim, J.-H. Jung, S. O. Hong and D.-W. Lee, Carbohydr. Polym., 2014, 103, 170-178.

33 Q.-L. Ma, L.-Z. Zhao, R.-R. Liu, B.-Q. Jin, W. Song, Y. Wang, Y.-S. Zhang, L.-H. Chen and Y.-M. Zhang, Biomaterials, 2014, 35, 9853-9867.

34 S. Franz, S. Rammelt, D. Scharnweber and J. C. Simon, Biomaterials, 2011, 32, 6692-6709.

35 G. Bhardwaj, H. Yazici and T. J. Webster, Nanoscale, 2015, 7, 8416-8427.

36 B. Jacob, I. S. Park, J. K. Bang and S. Y. Shin, J. Pept. Sci., 2013, 19, 700-707.

37 B. e. Nie, H. Ao, C. Chen, K. Xie, J. Zhou, T. Long, T. Tang and B. Yue, $R S C A d v ., 2016,6$, 46733-46743.

38 M. Godoy-Gallardo, C. Mas-Moruno, K. Yu, J. M. Manero, F. J. Gil, J. N. Kizhakkedathu and D. Rodriguez, Biomacromolecules, 2015, 16, 483-496.

39 A. Rodríguez-Cano, P. Cintas, M.-C. Fernández-Calderón, M.-Á. Pacha-Olivenza, L. Crespo, L. Saldaña, N. Vilaboa, M.-L. González-Martín and R. Babiano, Colloids Surf., B, 2013, 106, 248-257.

40 M. Riool, L. de Boer, V. Jaspers, C. M. van der Loos, W. J. B. van Wamel, G. Wu, P. H. S. Kwakman and S. A. J. Zaat, Acta Biomater., 2014, 10, 5202-5212.

41 L. Zhao, P. K. Chu, Y. Zhang and Z. Wu, J. Biomed. Mater. Res., Part B, 2009, 91, 470-480.

42 Z. Sheikh, P. J. Brooks, O. Barzilay, N. Fine and M. Glogauer, Materials, 2015, 8, 5671-5701.

43 M. Catauro, F. Bollino, F. Papale, C. Ferrara and P. Mustarelli, Mater. Sci. Eng., C, 2015, 55, 118-125. 
44 J. Chen, J. Cao, J. Wang, M. F. Maitz, L. Guo, Y. Zhao, Q. Li, K. Xiong and N. Huang, J. Colloid Interface Sci., 2012, 368, 636-647.

45 V. Escamilla-Rivera, M. Uribe-Ramírez, S. González-Pozos, O. Lozano, S. Lucas and A. De Vizcaya-Ruiz, Toxicol. Lett., 2016, 240, 172-184.
46 S. Lee, J. Choi, S. Shin, Y.-M. Im, J. Song, S. S. Kang, T.-H. Nam, T. J. Webster, S.-H. Kim and D. Khang, Acta Biomater., 2011, 7, 2337-2344.

47 K. M. Hotchkiss, G. B. Reddy, S. L. Hyzy, Z. Schwartz, B. D. Boyan and R. Olivares-Navarrete, Acta Biomater., 2016, 31, 425-434. 